\title{
Unintended Movement
}

National Cancer Institute

\section{Source}

National Cancer Institute. Unintended Movement. NCI Thesaurus. Code C62814.

Movement of the device to an unintended location within the body. 